\title{
Relation between Neutrophil to Lymphocyte Ratio, Platelet to Lymphocyte Ratio and Cerebral Infarct Volume in patients with Ischemic Stroke
}

\author{
Avni Uygar Seyhan*, Erdal Yilmaz, Semih Korkut
}

Received: 11 December 2020 / Accepted: 27 December 2020 / Published online: 20 January 2021

(C) The Author(s) 2021. This article is published with open access at https://journal.astes.org.al

\begin{abstract}
Objectives: The activation of inflammatory processes in ischemic stroke might be important for the pathophysiological processes of ischemic stroke. The correlation between the Neutrophil to Lymphocyte Ratio (NLR), Platelet to Lymphocyte Ratio (PLR), and stroke volume is increasingly being recognized as a prognostic biomarker for patient outcome after an ischemic stroke incidence. This retrospective study aimed at determining if there is existed a correlation between the NLR, PLR, and stroke volume in patients presenting with ischemic stroke at the emergency department. The results will aid emergency doctors to gain an understanding of how to rapidly identify the most high-risk patients based on the NLR and taPLR obtained from routine blood tests permitting rapidly therapeutic interventions and better long-term survival outcomes for patients with ischemic strokes.

Methods: A retrospective study was performed between May 2016-2019. Patients 18 years and older of both genders presented to the emergency department with symptoms of ischemic stroke with a time of onset of symptoms within the last 24 hours of whom complete blood count $(\mathrm{CBC})$ tests were conducted and ischemic stroke was confirmed by Diffusion-weighted Magnetic Resonance Imaging (DWI-MRI) were enrolled. NLR and PLR were calculated from CBC tests and ischemic stroke volume was measured in $\mathrm{mm}^{3}$ using Manual Segmentation Process.

Results: A total of 489 patients were enrolled in this study of which $266(54.5 \%)$ were male and 223 (45.6\%) were female. Was observed a statistically significant correlation between the ischemic stroke volume and NLR while no significant correlation was observed between stroke volume and PLR.

Conclusion: There is a significant weak positive relationship between NLR and ischemic stroke volume. NLR is significantly correlated with cerebral ischemic stroke volume and thus can be utilized as a guide by emergency doctors in the emergency department to predict the severity and the outcome of patients diagnosed with ischemic stroke.
\end{abstract}

Keywords: ischemic stroke, Neutrophil, Lymphocyte, Platelet, Emergency

\section{Introduction}

Strokes are one of the most common causes for mortality and the third leading cause of morbidity worldwide. Among all strokes, ischemic stroke accounts for approximately $80 \%$ to $85 \%$ of mortality in patients, and is characterized by the disruption of cerebral blood flow [1]. It has been

Original article, no submission or publication in advance or in parallel

* Corresponding author:

Avni Uygar SEYHAN MD, Ph.D.

$\bowtie$ uygarseyhan@gmail.com

“Kartal Dr. Lutfi Kirdar City Hospital “ TURKEY. suggested that inflammatory response is implicated in all stages of ischemic stroke. Thrombolytic therapy is the treatment of choice for ischemic stroke. However, risks associated with this form of therapy is intracranial bleeding and as such, only a limited number of patients will benefit from thrombolytic therapy [2]. To this effect, it is of utmost important to determine risk-stratification biomarkers that can be utilized for the prognostic significance after an acute ischemic stroke event. The most commonly utilized markers for patient outcome after an episode of ischemic stroke are the stroke volume and the National Institute of Health Stroke Scale (NIHSS) [3]. Atherosclerosis has been established to occur due to inflammatory processes and several inflammatory biomarkers have been identified that can be utilized to predict patient outcome after the occurrence of an ischemic stroke [4]. Elevated NLR has been associated 
with an adverse outcome after a cardiovascular event or surgery as well as an acute kidney injury and remote diffusion weighted image lesions in acute intracranial haemorrhage [5]. Increased NLR levels have additionally been observed post-operationally in diabetes patients after metabolic surgery and studies conducted by Luo et al. (2020) demonstrated that NLR can serve as a prognostic marker in acute minor stroke or transient ischemic attack with elevated levels of NLR being correlated with short term unfavourable functional outcome in patients diagnosed with transient ischemic stroke [6]. Studies conducted by Fang et al. (2017) demonstrated that elevated NLR is of prognostic significance and can predict the survival outcome of patients that have been diagnosed with non-atrial fibrillation caused by an ischemic stroke [4]. A meta-analysis conducted by Song et al. (2019) demonstrated that higher NLR levels were correlated with an increased risk of ischemic stroke and an unfavourable outcome and increased mortality 3 months after the ischemic event [7]. To this effect this study will aim at determining the correlation between NLR, PLR and stroke volume in patients that presented at the emergency department with an ischemic stroke event.

\section{Materials, method, patient enrolment and data collection:}

This retrospective observational study was carried out in the Emergency Department of a tertiary care teaching hospital between May 2016-2019. Patients 18 years and older of both genders diagnosed with ischemic stroke were retrospectively identified based on their medical records. Patients presented to the emergency department with symptoms of ischemic stroke with time of onset of symptoms within the last 24 hours of whom complete blood count (CBC) tests were conducted and ischemic stroke was confirmed by Diffusion weighted magnetic resonance imaging (DWI-MRI) were enrolled. Patients with haematological malignancies, patients with systemic infections or sepsis, patients on steroid therapy and patients receiving chemotherapy or radiotherapy for malignancies were excluded from this study. After excluding 84 patients for the reasons already mentioned a total number of 489 patients were enrolled in this study with 266 males and 223 females. The parameters that has been studied are the Neutrophils to Lymphocyte Ratio (NLR), Platelet to Lymphocyte Ratio (PLR), ischemic stroke volume measured by $\left(\mathrm{mm}^{3}\right)$, the anatomical location of the stroke (anterior vs posterior circulation stroke) and the gender of the patient. The patient's parameters were revised from hospital medical electronic records. DWI MRI's were revised from INFINITT PACS 7.0 software. The stroke volume was analysed by Manual Segmentation Process using ITK- SNAP 3.8.0 software and this has been done by a well-trained radiologist. SPSS Statistics 23.0 package program was used for statistical analysis. This study was approved in 10.07.2019 by the local ethics committee (Ethics Committee Ruling number (2019/514/157/1).

\section{Results}

1. Determination of a correlation between ischemic stroke volume, NLR and PLR:

A total of 489 patients were enrolled in this study of which $266(54.5 \%)$ were male and $223(45.6 \%)$ were female. Anterior circulation stroke was identified in 318 (65\%) of the study participants while a posterior circulation stroke was identified in 171 (35\%) of the participants with both males and females in each group. A mean stroke volume of $569.28 \pm 777.29$ was recorded in all 489 patients with a minimum of 0.001 and a maximum of 2249.0. A mean NLR (minimum $=0.67$ and maximum=24.67) of $4.12 \pm 3.42$ and a mean PLR (minimum $=0.01$ and maximum $=1098.57$ ) $163.12 \pm 94.41$ was recorded for all patients as well. A summary of the results is shown in (Table 1). Kendall's Tau $b$ correlation analysis was performed to reveal the presence of the relationship between stroke volume and NLR and PLR variables due to the abnormal distribution of all variables. Kendall's Tau B correlation analysis revealed a negative weak relationship between stroke volume and NLR variables $(r=-0.106, p<0.05)$. This relationship between stroke volume and NLR is statistically significant. The correlation coefficient between stroke volume and PLR variables was found to be $r=-0.055$ and this relationship was not statistically significant $(\mathrm{p}>0.05)$ (Table 2$)$.

\begin{tabular}{|l|l|c|l|l|c|}
\hline \multicolumn{6}{|c|}{ Basic Statistics } \\
\hline & $\mathrm{N}$ & Minimum & Maximum & Mean & Std. Deviation \\
\hline $\begin{array}{l}\text { Stroke } \\
\text { volume }\end{array}$ & 489 & 0.001 & 2249.0 & 569.2883 & 777.29 \\
\hline NLR & 489 & 0.67 & 24.67 & 4.12 & 3.42 \\
\hline PLR & 489 & 0.01 & 1098.57 & 163.239 & 94.41 \\
\hline
\end{tabular}

Table 1: The mean of the Stroke volume, NLR and PLR as a function of the minimum and maximum values in 489 patients.

\begin{tabular}{|l|l|c|c|}
\hline & & NLR & PLR \\
\hline $\begin{array}{l}\text { Stroke } \\
\text { volume }\end{array}$ & $\begin{array}{l}\text { Kendall's Taub } \\
\text { Correlation Coefficient }\end{array}$ & $-.106^{*}$ & -.055 \\
\cline { 2 - 4 } & P value (2-way) & .002 & .100 \\
\cline { 2 - 4 } & $\mathrm{N}$ & 489 & 489 \\
\hline
\end{tabular}

Table 2: Kendall's Tau b correlation analysis to determine the relationship between stroke volume, NLR and PLR variables $(*$ denoted statistical significance at levels $p<0.05)$

\section{2- Correlation between stroke volume, NLR and PLR} as a function of the anterior and posterior circulation.

A measure of the stroke volume in terms of the anterior and posterior circulation demonstrated that a mean stroke volume of $395 \pm 722.22$ for the anterior circulation was calculated for 318 patients while for 171 patients, the mean stroke volume for posterior circulation was estimated at $892.099 \pm 774.94519$ (total $=569.28 \pm 777.29468)$. The NLR in function of anterior circulation was $4.0063 \pm 3.17691$ 
while the mean posterior circulation was recorded as $4.3531 \pm 3.84544$ with a total of $4.1276 \pm 3.42563$ for all 489 patients being recorded. In terms of the PLR, a mean anterior circulation of $163.302 \pm 96.26$ was calculated for 318 patients while a mean posterior circulation of $163.1223 \pm 91.14$ was recorded for 171 patients (total $=163.29 \pm 94.41$ ). Results shown in (Table 3).

\begin{tabular}{|l|l|l|l|l|}
\hline \multicolumn{2}{|l|}{ Circulation Type } & $\begin{array}{l}\text { Stroke } \\
\text { volume } \mathrm{mm}^{3}\end{array}$ & NLR & PLR \\
\hline \multirow{2}{*}{$\begin{array}{l}\text { Anterior } \\
\text { Circulation }\end{array}$} & Mean & 395.7013 & 4.0063 & 163.3023 \\
\cline { 2 - 5 } & $\mathrm{N}$ & 318 & 318 & 318 \\
\cline { 2 - 5 } & Std. Deviation & 722.22478 & 3.17691 & 96.26835 \\
\hline $\begin{array}{l}\text { Posterior } \\
\text { Circulation }\end{array}$ & Mean & 892.0994 & 4.3531 & 163.1223 \\
\cline { 2 - 5 } & $\mathrm{N}$ & 171 & 171 & 171 \\
\cline { 2 - 5 } & Std. Deviation & 774.94519 & 3.84544 & 91.14514 \\
\hline Total & Mean & 569.2883 & 4.1276 & 163.2394 \\
\cline { 2 - 5 } & $\mathrm{N}$ & 489 & 489 & 489 \\
\cline { 2 - 5 } & Std. Deviation & 777.29468 & 3.42563 & 94.41467 \\
\hline
\end{tabular}

Table 3: The mean stroke volume, NLR and PLR as a function of the anterior and posterior circulation.

\section{a- Correlation between stroke volume of the anterior circulation, NLR and PLR.}

The relationship between stroke volume of anterior circulation, NLR and PLR was calculated and analyzed by calculating Kendall's Tau b correlation coefficients due to the persistence and abnormal distribution of the variables. The correlation coefficient between the posterior circulation stroke volume and NLR was estimated to be $\mathrm{r}=-0.101$ demonstrating a weak correlation between both variables which was statistically significant $(\mathrm{p}<0.05)$. Kendall's Tau $\mathrm{b}$ correlation coefficient indicating the direction and level of relationship between the anterior circulation stroke volume and PLR was found to be $r=-0.032$. The relationship between the anterior circulation stroke volume and PLR was not statistically significant $(\mathrm{p}>0.05)$ (Table 4)

\begin{tabular}{|c|c|c|c|}
\hline \multirow{4}{*}{$\begin{array}{l}\text { Anterior } \\
\text { Circulation } \\
\text { Stroke } \\
\text { volume }\end{array}$} & & NLR & PLR \\
\hline & $\begin{array}{l}\text { Kendall's Taub } \\
\text { Correlation Coefficient }\end{array}$ & $-.101 *$ & .032 \\
\hline & P value (2-way) & .020 & .457 \\
\hline & $\mathrm{N}$ & 317 & 317 \\
\hline
\end{tabular}

Table 4: Kendall's Tau b correlation coefficient between anterior circulation stroke volume and NLR and PLR. A* denoted statistical significance with $(p<0.05)$

b- Correlation between stroke volume of the posterior circulation, NLR and PLR

The relationship between stroke volume of posterior circulation, NLR and PLR was calculated and analyzed by calculating Kendall's Tau b correlation coefficients due to the persistence and abnormal distribution of the variables. The correlation coefficient between the posterior circulation stroke volume and NLR was estimated to be $\mathrm{r}=-0.147$. As such existed a weak, negative correlation between the posterior circulation stroke volume and NLR and this was statistically significant $(\mathrm{p}<0.05)$. The correlation coefficient between the posterior circulation stroke volume and PLR was found to be $\mathrm{r}=-0.087$ and it was not statistically significant $(\mathrm{p}>0.05)$ (Table 5).

\begin{tabular}{|c|c|c|c|}
\hline \multirow{4}{*}{$\begin{array}{l}\text { Posterior } \\
\text { Circulation } \\
\text { Stroke } \\
\text { volume }\end{array}$} & & NLR & PLR \\
\hline & $\begin{array}{l}\text { Kendall's Taub } \\
\text { Correlation Coefficient }\end{array}$ & $-.147 *$ & -.087 \\
\hline & $\mathrm{P}$ value (2-way) & .006 & .105 \\
\hline & $\mathrm{N}$ & 174 & 174 \\
\hline
\end{tabular}

Table 5: Kendall's Tau b correlation coefficient between posterior circulation stroke volume and NLR and PLR. A* denoted statistical significance with $(p<0.05)$

\section{Discussion}

In this retrospective study of patients presenting with ischemic stroke symptoms at the emergency department, we demonstrated that there is a significantly negative correlation between stroke volume and NLR. It was observed a statistically significant correlation between the stroke volume (in anterior and posterior stroke circulation) and NLR. The correlation coefficient of Kendall's Tau b, which shows the relationship between the anterior and posterior circulation stroke volume and NLR, denoted a significantly weak level of correlation which is significant. As NLR increases, stroke volume decreases or the reverse holds true wherein the, stroke volume increases while NLR decreases. On the other hand, it was observed that the relationship between the stroke volume (in anterior and posterior circulation stroke) and PLR was not statistically significant. Increased NLR has been reported to be correlated to poor patient outcome and a significant predictor of short-term mortality in patients presenting with an ischemic stroke episode [8-12]. Several studies have established the function of the inflammatory system in the different stages of acute ischemic stroke. During an ischemic episode, the release of proinflammatory cytokines and the recruitment of immune cells at ischemic sites might be responsible for secondary brain lesions [13]. Neutrophils are the primary immune cells that are most often recruited to brain lesions. These neutrophils exacerbate brain injuries due to their secretion of proteolytic enzymes such as acid phosphatase or reactive oxygen species (ROS) [14]. The relationship between increased NLR and patient mortality might be due to the fact that the NLR might be elevated due to the activation of an inflammatory response to an ischemic stroke episode or it can act as a predictor for the extent and severity of neurological damage due to the presence of necrotic tissue. The early stratification of patients diagnosed with ischemic stroke is essential for the determination of the best course of treatment for patients leading to improved patient outcome. NLR is an efficient biomarker in terms of stroke volume and 
can be utilized to predict patient outcome after an ischemic episode. A higher NLR might be indicative of the severity of an ischemic episode thus highlighted the importance of this biomarker for risk stratification. We thus suggest that NLR might be utilized to identify and stratify high-risk ischemic stroke patients that are in need of endovascular interventions.

\section{Limitation:}

There existed several limitations in this study of which was the lack of information on the long-term survival of the patients included in this study. Secondly, despite the fact that patients with active infections and other underlying conditions such as patients with hematological malignancies, patients with sepsis and patients on steroids were excluded from the study, many other diseases that might activate inflammatory processes might have not been considered. Studies with long-term follow-up are thus needed to establish the prognostic significance of NLR in patients diagnosed with ischemic stroke.

\section{Conclusion:}

In conclusion NLR is significantly correlated with stroke volume and thus might be utilized as a guide in deciding the therapeutic interventions that are required to treat the patient.

COI Statement: This paper has not been submitted in parallel. It has not been presented fully or partially at a meeting or podium or congress. It has not been published nor submitted for consideration beforehand.

This research received no specific grant from any funding agency in the public, commercial, or nonprofit sectors. There are no relevant or minor financial relationships from authors, their relatives or next of kin with external companies.

Disclosure: The authors declared no conflict of interest. No funding was received for this study.

\section{References}

1. Chugh, C. (2019). Acute Ischemic Stroke: Management Approach. Indian Journal of Critical Care Medicine: Peer-Reviewed, Official Publication of Indian Society of Critical Care Medicine, 23(Suppl 2), S140-S146. https://doi. org/10.5005/jp-journals-10071-23192

2. Xiong, Y., Manwani, B., \& Fisher, M. (2019). Management of Acute Ischemic Stroke. The American Journal of Medicine, 132(3), 286-291. https://doi.org/10.1016/j. amjmed.2018.10.019

3. Mahdy, M. E., Ghonimi, N. A., Elserafy, T. S., \& Mahmoud, W. (2019). The NIHSS score can predict the outcome of patients with primary intracerebral hemorrhage. The Egyptian Journal of Neurology, Psychiatry and Neurosurgery, 55(1), 21. https://doi.org/10.1186/s41983-019-0056-0
4. Fang, Y.-N., Tong, M.-S., Sung, P.-H., Chen, Y.-L., Chen, C.-H., Tsai, N.-W., Huang, C.-J., Chang, Y.-T., Chen, S.F., Chang, W.-N., Lu, C.-H., \& Yip, H.-K. (2017). Higher neutrophil counts and neutrophil-to-lymphocyte ratio predict prognostic outcomes in patients after non-atrial fibrillationcaused ischemic stroke. Biomedical Journal, 40(3), 154-162. https://doi.org/https://doi.org/10.1016/j.bj.2017.03.002

5. Li, J.-W., Xu, Y.-Y., Chen, Y.-J., Fan, W.-W., Xu, X.-H., Cai, J.-S., Tong, L.-S., \& Gao, F. (2020). Early elevated neutrophilto-lymphocyte ratio associated with remote diffusionweighted imaging lesions in acute intracerebral hemorrhage. CNS Neuroscience \& Therapeutics, 26(4), 430-437. https:// doi.org/10.1111/cns.13249

6. Luo, Y., Xia, L., Li, Z., Pi, D., Tan, X., \& Tu, Q. (2020). Early neutrophil-to-lymphocyte ratio is a prognostic marker in acute minor stroke or transient ischemic attack. Acta Neurologica Belgica. https://doi.org/10.1007/s13760-020-01289-3

7. Song, S.-Y., Zhao, X.-X., Rajah, G., Hua, C., Kang, R.-J., Han, Y.-P., Ding, Y.-C., \& Meng, R. (2019). Clinical Significance of Baseline Neutrophil-to-Lymphocyte Ratio in Patients With Ischemic Stroke or Hemorrhagic Stroke: An Updated Meta-Analysis. Frontiers in Neurology, 10, 1032. https://doi. org/10.3389/fneur.2019.01032

8. Giede-Jeppe, A., Madžar, D., Sembill, J. A., Sprügel, M. I., Atay, S., Hoelter, P., Lücking, H., Huttner, H. B., \& Bobinger, T. (2019). Increased Neutrophil-to-Lymphocyte Ratio is Associated with Unfavorable Functional Outcome in Acute Ischemic Stroke. Neurocritical Care. https://doi.org/10.1007/ s12028-019-00859-5

9. Kocaturk, O., Besli, F., Gungoren, F., Kocaturk, M., \& Tanriverdi, Z. (2019). The relationship among neutrophil to lymphocyte ratio, stroke territory, and 3-month mortality in patients with acute ischemic stroke. Neurological Sciences, 40(1), 139-146. https://doi.org/10.1007/s10072-018-3604-y

10. Suh, B., Shin, D. W., Kwon, H.-M., Yun, J. M., Yang, H.-K., Ahn, E., Lee, H., Park, J. H., \& Cho, B. (2017). Elevated neutrophil to lymphocyte ratio and ischemic stroke risk in generally healthy adults. PloS One, 12(8), e0183706e0183706. https://doi.org/10.1371/journal.pone.0183706

11. Wang, L., Song, Q., Wang, C., Wu, S., Deng, L., Li, Y., Zheng, L., \& Liu, M. (2019). Neutrophil to lymphocyte ratio predicts poor outcomes after acute ischemic stroke: A cohort study and systematic review. Journal of the Neurological Sciences, 406. https://doi.org/10.1016/j.jns.2019.116445

12. Zhang, R., Wu, X., Hu, W., Zhao, L., Zhao, S., Zhang, J., Chu, Z., \& Xu, Y. (2019). Neutrophil-to-lymphocyte ratio predicts hemorrhagic transformation in ischemic stroke: A metaanalysis. Brain and Behavior, 9(9), e01382-e01382. https:// doi.org/10.1002/brb3.1382

13. Świtońska, M., Słomka, A., Korbal, P., Piekuś-Słomka, N., Sinkiewicz, W., Sokal, P., \& Żekanowska, E. (2019). Association of Neutrophil-to-Lymphocyte Ratio and Lymphocyte-to-Monocyte Ratio with Treatment Modalities of Acute Ischaemic Stroke: A Pilot Study. In Medicina (Vol. 55, Issue 7). https://doi.org/10.3390/medicina55070342

14. Qun, S., Tang, Y., Sun, J., Liu, Z., Wu, J., Zhang, J., Guo, J., Xu, Z., Zhang, D., Chen, Z., Hu, F., Xu, X., \& Ge, W. (2017) Neutrophil-To-Lymphocyte Ratio Predicts 3-Month Outcome of Acute Ischemic Stroke. Neurotoxicity Research, 31(3), 444-452. https://doi.org/10.1007/s12640-017-9707-z 